\title{
Desenvolvendo atitudes, conhecimentos e habilidades dos estudantes de medicina na atenção em saúde de pessoas surdas
}

Luiza Santos Moreira da Costa ${ }^{1}$ Natália Chilinque Zambão da Silva

\section{Introdução}

Pelo fato da surdez não demandar uma acessibilidade facilmente identificável, como é o caso de pessoas com deficiência física, existe uma falsa impressão que os serviços e profissionais de saúde estariam atendendo às necessidades desse grupo de forma satisfatória. Pesquisas em diversos países demonstram o contrário e apontam a vulnerabilidade das pessoas surdas no aspecto da saúde (World Health Organization, 2011; Costa et al., 2009). Surdos que se comunicam através da língua de sinais acabam procurando os serviços de saúde com menos frequência e aguardam a disponibilidade de um intérprete ou pessoa próxima que possa desempenhar esse papel, adiando, muitas vezes, a visita ao profissional de saúde (Chaveiro, 2007). Obstáculos à comunicação interferem na capacidade do sistema de saúde em atender, de forma adequada, as necessidades de saúde deste grupo (Chaveiro, 2007; Zazove, Doukas, 1994), incluindo o risco de erros no diagnóstico e no tratamento (U.S. Department of Justice, 2003). Muitos surdos não oralizados têm dificuldade em entender a medida do remédio ou o que significa "de hora em hora" (Costa et al., 2009); possuem menos informação que os ouvintes sobre doenças cardiovasculares (Margellos-Anast, Estarziau, Kaufman, 2006) e em relação ao HIV/Aids (Bat-Chava, Martin, Kosciw, 2005).

No Brasil, grande parte das pessoas surdas que se comunicam através de língua de sinais estuda em escolas especiais (o movimento de inclusão escolar é recente) e se relaciona socialmente nas Associações de Surdos, devido às barreiras linguísticas, o que acaba por gerar falta de convívio entre surdos e ouvintes. Esta segregação e o silêncio sobre como facilitar a comunicação entre médicos e pacientes surdos, durante a formação médica, levam a concepções errôneas desses profissionais sobre as pessoas surdas e suas formas de comunicação, tais como: a) a leitura labial é uma habilidade universal dos surdos; b) a escrita é sempre um método eficaz de se comunicar com pacientes surdos; e c) o intérprete de língua de sinais deve ser seu interlocutor (lezzoni et al., 2004; Zazove, Doukas, 1994). Mesmo um surdo oralizado (que se comunica através da fala e da leitura labial) precisa que se fale olhando para ele, articulando as palavras. Quanto ao uso da escrita para se comunicar com pacientes surdos, Barnett (2002) lembra que, apesar de a escrita representar um fator facilitador na

$$
\begin{array}{r}
\text { Elaborado com base } \\
\text { em proposta de inclusão } \\
\text { de temas ligados à } \\
\text { deficiência nos cursos de } \\
\text { graduação e pós- } \\
\text { graduação da } \\
\text { Universidade Federal } \\
\text { Fluminense. } \\
\text { 1 Departamento de } \\
\text { Saúde e Sociedade, } \\
\text { Universidade Federal } \\
\text { Fluminense. } \\
\text { Rua Marquês do } \\
\text { Paraná, 303. Hospital } \\
\text { Universitário Antonio } \\
\text { Pedro. Prédio anexo, } 3^{\circ} \\
\text { andar. Niterói, Rio de } \\
\text { Janeiro, RJ, Brasil. } \\
24.030-210 . \\
\text { luizacosta@id.uff.br } \\
2 \text { Graduada em } \\
\text { Medicina. }
\end{array}
$$


comunicação com pacientes surdos oralizados e com os que apresentam deficiência auditiva, é de pouca ajuda para os surdos pré-linguísticos (que ficaram surdos antes da aquisição da linguagem), sendo a língua de sinais sua primeira língua. Quanto à comunicação escrita, Almeida (2007) aponta que, embora a maioria dos surdos possa ler, eles nem sempre entendem o significado de diversas palavras, ou confundem o significado, em especial dos homônimos, como: partir (ir embora/dividir), além das muitas expressões idiomáticas. Menos de 10\% das mulheres surdas que participaram da investigação realizada em Chesire (Reino Unido), sobre desigualdades no acesso aos cuidados de saúde enfrentadas por mulheres surdas (Ubido, Huntington, Warburton, 2002), informaram que costumam compreender tudo o que os médicos Ihes dizem quando vão sozinhas às consultas.

Como resultado desse desconhecimento em relação à pessoa surda, o profissional de saúde acaba falhando em ver o paciente surdo como alguém que poderia contribuir no processo do cuidado da sua própria saúde; isto é, ignora o indivíduo enquanto fonte potencial de informação clínica (Eddey, Robey, 2005). Os surdos avaliam que alguns médicos acreditam que não adiantaria explicar porque o surdo não seria capaz de entender (Costa et al., 2009). Muitas vezes, a comunicação durante toda a consulta se dá apenas entre o médico e o acompanhante. Em entrevista realizada com surdos com domínio apenas da língua de sinais como forma de se comunicar, um dos relatos exemplificou bem essa falta de autonomia e de independência do paciente surdo, que, além de depender de uma terceira pessoa para acompanhá-lo às consultas, não toma parte nas negociações terapêuticas:

Fui ao médico quando tinha problema dos rins. Não entendia nada do que o médico falava. Minha mãe teve que ir comigo para me ajudar. O médico falava e fiquei perdida; perguntava para minha mãe o que ele falou. Ela me dizia para esperar que em casa me contaria. Então, em casa, minha mãe me contou, mas resumidamente, e no consultório minha mãe e o médico falaram muito e ela só me contou muito pouco. (Costa et al., 2009, p.168)

Se, por um lado, os surdos que usam língua de sinais se sentem mais seguros com a presença de um(a) intérprete (Steinberg et al., 2002) durante as consultas e, mesmo, na sala de parto, por outro queixam-se de falta de privacidade com a presença do intérprete na consulta com um ginecologista.

Conversando com pessoas surdas, também ouvimos relatos de exemplos positivos, como o de uma surda bilíngue. O médico foi atencioso, falou devagar e, quando ela não entendia, o médico não se furtava a repetir ou à solicitação que escrevesse (Costa et al., 2009).

Visando alterar o cenário aqui apresentado, a partir do início deste século vem crescendo o número de experiências interessantes nas escolas médicas australianas, americanas, do Reino Unido, da África do Sul e da Croácia, dentre outras, buscando incluir temas ligados à deficiência nos currículos médicos. São realizadas atividades voltadas à promoção de atitudes positivas em relação à pessoa com deficiência, ao conhecimento a respeito desse grupo, e/ou desenvolvimento de habilidades de comunicação ou de exame clínico de pacientes com sequela de lesão medular, e produção de material de apoio. Pessoas com deficiência, seus familiares e organizações que lutam por seus direitos vêm participando do planejamento da inclusão curricular. Seminários; aulas formais, vivências; visitas domiciliares; e participação de pessoas com deficiência sendo entrevistadas pelos estudantes; atuando como tutores ou pacientes-padronizados, estão entre as abordagens mais frequentes (Duggan et al., 2009; Symons, McGuigan, Akl, 2009; Tracy, Iacono, 2008; Eddey, Robey, 2005; Martin et al., 2005; Byron et al., 2005; Minihan et al., 2004; Vlak et al., 2004; Thistlethwaite, Ewart, 2003; Sabharwal, Brownell, 2001; Byron, Dieppe, 2000; Sabharwal, Sebastian, Lanquette, 2000; Henley, 1999; Conill, 1988; Mitchell et al., 1984).

A importância do ensino de comunicação nos cursos de graduação em medicina como campo de conhecimento a ser contemplado nos projetos pedagógicos da formação de futuros médicos já é reconhecida. Justifica-se pelos resultados de pesquisas sobre a relação médico-paciente, e de importantes discussões, especialmente em fóruns internacionais, e das recomendações das novas Diretrizes Curriculares Nacionais (Rossi, Batista, 2006), embora ainda não incluam as particularidades da comunicação com pessoas não verbais. Os mesmos autores apontaram ainda, em sua investigação sobre o ensino da comunicação em Medicina, que a atividade prática junto ao paciente desempenha um 
papel de destaque no ensino/aprendizagem das habilidades de comunicação, e que a observação de atitudes e comportamentos no cotidiano do ensino, seja de professores ou de outros médicos em atividade, notadamente no internato, seria a principal responsável pela aprendizagem desta habilidade pelos alunos. Mas, no caso da comunicação com pessoas com deficiência, se os médicos e professores não tiveram, em sua formação, esse conteúdo, que modelos os estudantes estariam observando?

No Brasil já existem esforços em chamar a atenção para essa lacuna (Costa et al., 2009; Costa, Botelho, Souza, 2008; Silva, Costa, 2008; Vieira et al., 2008; Chaveiro, 2007; Chelini et al., 2006; Costa, 2005; Ferreira, Koifman, Costa, 2005). Na Universidade Federal Fluminense (UFF), desde o ano de 2004, a atenção integral à saúde da pessoa com deficiência e sua inclusão social passaram a fazer parte das disciplinas Trabalho de Campo Supervisionado I e Saúde e Sociedade III, dirigidas, respectivamente, aos $2^{\circ}$. e $3^{\circ}$. períodos do curso médico. O presente relato refere-se à inclusão do tema Deficiência na disciplina Saúde e Sociedade III, desde 2007, inaugurando o treinamento de habilidades de comunicação considerando pessoas surdas.

O objetivo deste artigo é chamar a atenção de profissionais de saúde e docentes que atuam na formação de recursos humanos na área da saúde, trazendo visibilidade para a pessoa com deficiência no caso específico, para a pessoa surda - nos currículos do curso médico, apresentando o relato de experiência de inclusão de atividades dirigidas a capacitar futuros médicos para se comunicarem com pessoas surdas. Partimos dos seguintes pressupostos que orientaram e justificaram a inclusão proposta:

- A Comunicação Competente faz parte do Aconselhamento em Saúde preconizado pelo Ministério da Saúde (Filgueiras, Deslandes, 1999). Em relação às pessoas com deficiência, significa: comunicar-se com pacientes que apresentem déficits na comunicação verbal; evitar fala infantilizada; compreender os valores e necessidades das pessoas com deficiência, e sentir-se confortável com pacientes, inclusive com deficiências complexas, entre outros (Eddey, Robey, 2005);

- A assistência integral à saúde da pessoa com deficiência não se restringe à prestada em instituições específicas de reabilitação, mas ao atendimento na rede de serviços, nos diversos níveis de complexidade e especialidades médicas, segundo a Política Nacional de Saúde da Pessoa com Deficiência (Brasil, 2006; 2002);

- A capacitação de recursos humanos para o desenvolvimento das ações decorrentes dessa Política inclui a necessidade de que sejam incorporadas disciplinas e conteúdos de reabilitação e atenção à saúde das pessoas com deficiência nos currículos de graduação da área da saúde (Brasil, 2002). As Diretrizes Curriculares Nacionais do Curso de Graduação em Medicina apontam que a formação do médico tem por objetivo dotar o profissional de conhecimentos requeridos para o exercício de competências e habilidades que incluem, no item Comunicação, tanto a comunicação verbal quanto não verbal, permitindo comunicar-se adequadamente com os pacientes e seus familiares; e o uso de técnicas apropriadas de comunicação para informar e educar seus pacientes, familiares e comunidade em relação à promoção da saúde, prevenção, tratamento e reabilitação das doenças (Brasil, 2001);

- Filosofia do Movimento de Vida Independente, segundo a qual a pessoa com deficiência é que deve ter o controle da situação e da sua vida; ter o direito de realizar escolhas (Paula, 2008);

- "Nada sobre nós sem nós" - significa que nenhum resultado a respeito das pessoas com deficiência deverá ser gerado sem a plena participação das próprias pessoas com deficiência (Sassaki, 2007).

\section{Metodologia das atividades}

Na disciplina Saúde e Sociedade III, dirigida ao terceiro período do curso médico da UFF, entre 2007 e 2010, participaram, das aulas de Comunicação competente, pessoas surdas e com sequela de encefalopatia crônica da infância (disartria e espasticidade). Serão aqui descritas as atividades que tiveram por objetivo: promover o conhecimento sobre aspectos relacionados à comunicação de pessoas surdas; desenvolver habilidades de comunicação com surdos que se comunicam de diferentes formas, e promover atitudes positivas em relação a pessoas com deficiência, em especial, pessoas surdas. 


\section{Descrição das atividades}

Nos sete semestres, 553 estudantes participaram dessas atividades $\left(75\right.$ no $1^{\circ}$ semestre e 78 no $2^{\circ}$ de 2007; 84 no $1^{\circ}$. semestre e 78 no $2^{\circ}$ de $2008 ; 83$ no $1^{\circ}$ semestre e 78 no $2^{\circ}$ de 2009 ; e 77 no $1^{\circ}$ semestre de 2010). O tema ocupou duas aulas, com duração de três horas cada uma. Na primeira aula, teórica, foram discutidos os textos: Considering the Culture of Disability in Cultural Competence (Eddey, Robey, 2005); Encontro do Paciente Surdo que usa Língua de Sinais com os Profissionais da Saúde (Chaveiro, 2007), e A Língua Brasileira de Sinais - Libras (Felipe, 2005). Nesse mesmo dia, também foram ensinados alguns sinais relativos ao corpo humano, sexo e doenças sexualmente transmissíveis, apresentados em vídeo. A segunda aula foi ocupada com vivência planejada por duas professoras de Biologia, ouvintes, e três profissionais surdos do Núcleo de Orientação à Saúde do Surdo, do Instituto Nacional de Educação de Surdos, que também participaram das dramatizações. A Associação de Pais e Amigos dos Deficientes da Audição de Niterói disponibilizou tradutor/intérprete de Língua Brasileira de Sinais (Libras), que, além de intermediar a comunicação entre a turma e os profissionais surdos, participou da dramatização. Foram propostas quatro situações de busca por atendimento médico, de acordo com a forma de comunicação do paciente: 1) surdo oralizado; 2) surdo que se comunica através da Libras acompanhado de intérprete; 3) outro que vai à consulta sozinho; e 4) surdo que não aprendeu Libras, nem português, não sabe fazer leitura labial e não fala.

A dramatização começa com uma "paciente" surda sentada no espaço representando a sala de espera. O aluno, voluntário no papel de médico, não sabe ainda a forma de comunicação da "paciente". Os próprios surdos escolheram os temas da consulta: busca por método anticoncepcional, dengue, DST e sintomas gastrointestinais, chamando a atenção, durante cada consulta, para termos de difícil compreensão pelos surdos. Apesar de apenas quatro estudantes de cada turma terem participado diretamente da experiência enquanto "médicos", a partir das dificuldades encontradas para se comunicar com o "paciente" surdo, os demais colegas de turma propunham soluções.

Ao final de cada "consulta", foram apontadas e discutidas as dificuldades sentidas pelos "pacientes" e pelos "médicos", assim como formas de reduzi-las. Como fechamento da atividade, os estudantes conversaram com os convidados surdos, conhecendo um pouco sobre suas vidas.

Com o objetivo de avaliar o que representou para os estudantes esse contato direto com tutores surdos e realizar os ajustes necessários, solicitamos que cada aluno, individualmente, escrevesse, de forma livre e anônima, uma avaliação dessa atividade, a ser entregue na aula seguinte. Será apresentada aqui a análise das avaliações dos estudantes do $1^{\circ}$. e $2^{\circ}$. semestres de 2008 , e do $1^{\circ}$. semestre de 2009, que puderam ser recuperadas a partir de arquivo digital, ou seja, de 245 estudantes.

Optou-se pela análise qualitativa dos dados através da técnica de análise categorial (Gomes, 1997; Bardin, 1977). A partir da leitura flutuante das avaliações, foram destacados temas organizados nas categorias: Atitudes; Habilidades; e Conhecimentos - tomando-se como referência os três grandes objetivos da proposta de inclusão curricular de ensino médico sobre como cuidar de pessoas com deficiência, na escola médica da Universidade de Búfalo (Symons, McGuigan, Akl, 2009).

\section{Resultados}

As categorias e subcategorias serão apresentadas utilizando-se trechos dos relatos dos estudantes para exemplificá-las:

\section{Atitudes}

Esta categoria traduz a forma como a pessoa surda é vista: desenvolvimento de um olhar para a pessoa surda, vendo a pessoa para além da surdez; respeito e valorização dos direitos e desejos dessas pessoas; e exame de suas próprias atitudes a respeito da surdez.

. A pessoa surda sob um novo olhar: 
"Respeitando-se as peculiaridades do paciente, a surdez deixa seu caráter deficitário e assume apenas uma característica diferencial".

"A aula foi importante para quebrar a premissa de que os surdos são 'coitados' ou dignos de pena. O que pudemos ver é que eles são pessoas normais que levam uma vida normal, mas apresentam uma limitação que, muitas vezes, não é respeitada pela sociedade".

"Esta 'aula prática' é muito importante porque tira a idéia de que o fato de a pessoa ser surda limita sua capacidade de entendimento."

\section{. Colocar-se no lugar do outro:}

“Na simulação não tive ajuda para me expressar e me vi numa situação semelhante a que a pessoa surda tem em meio aos ouvintes - tentar me comunicar, me expressar, e não conseguir."

. Visibilidade para a pessoa surda:

"De imediato fiquei muito surpresa com o tema, pois nunca tinha atentado para as necessidades da pessoa surda. Além disso, não havia pensado que teria que me comunicar com essas pessoas".

. Encontro com o diferente:

"Acredito que para o sucesso da relação futuro médico - pessoa com deficiência, seja necessária essa troca mútua de opiniões e experiências, como vimos nessas aulas, para que possamos perder o medo de enfrentar o que é 'novo' e diferente para nós no dia-a-dia."

\section{Habilidades}

Refere-se à aquisição de recursos técnicos; de orientações práticas à comunicação, aplicáveis também em outras situações. Apresentar atitudes positivas em relação ao paciente surdo não basta para que a comunicação flua. É preciso que os estudantes aprendam como fazê-lo.

. Percepção das habilidades adquiridas:

"Aprendi que o profissional de saúde não precisa dominar a língua de sinais para que consiga se comunicar com o paciente. Basta que este se empenhe, seja paciente e utilize todo tipo de recurso que possuir (mímica, falar articulando as palavras adequadamente, escrever de modo simples, usar figuras) para que possa passar a mensagem de modo adequado à necessidade do paciente".

"Através desta experiência conseguimos ter um pouco de contato com as dificuldades dos surdos e do desafio que é estabelecer uma boa relação médico-paciente nessas circunstâncias. Dentre elas, a que mais chamou a minha atenção foi aquela em que o intérprete estava presente, pois neste momento, há o risco de a atenção e a comunicação se estabelecerem entre o médico e o intérprete, e não com o paciente".

. Aprender não apenas o que fazer, mas como fazer:

"A idéia de levar surdos para a aula é excelente porque imaginar o que o médico deve fazer durante uma anamnese com um surdo pode ser uma tarefa simples, mas ter a oportunidade de praticar isso é essencial para uma boa formação médica." 
"Com as dramatizações, mais do que novas informações, fomos desafiados a aplicar o que havíamos discutido ou o que pensávamos saber. Dessa forma fomos obrigados a criar outros mecanismos de comunicação quando o que conhecíamos não foi suficiente; situação esta que acontecerá no cotidiano da prática médica".

- Mudança de ideias equivocadas:

"Gostei de saber também que não é todo surdo que faz leitura labial. Antes, pensava que todos eram capazes de fazê-lo."

. Semelhança com outras situações:

"Há uma nítida dificuldade de qualquer pessoa em se comunicar com pessoas surdas; é como se estivesse num país estrangeiro, em uma língua que nem conhecemos. Foi essa a sensação sentida por parte do médico e do paciente também."

\section{Conhecimentos}

Essa categoria diz respeito à construção de um conhecimento geral sobre surdez: frequência e causas mais comuns; impacto da surdez no indivíduo e em sua família; e consequências de entraves na comunicação médico-paciente. Nessa categoria, a ênfase dos estudantes recaiu mais na forma como os conhecimentos foram adquiridos do que no conteúdo.

. Risco de atendimento com entraves na comunicação:

“Uma simples consulta médica, sem a devida comunicação, acaba sendo desrespeitosa ao paciente, além de ser prejudicial no caso de indicação de como usar o medicamento poder ser entendida da maneira errada".

. Complementação entre aula teórica e prática:

"A leitura do artigo e a aula teórica nos muniram de conhecimento básico sobre como proceder no atendimento, enquanto a dinâmica com os convidados apresentou as dificuldades na prática."

. Aprender com o real:

"Fico feliz por Saúde e Sociedade ter trazido para dentro da sala pessoas reais, com problemas verdadeiros, ao invés de propor uma fácil dinâmica de teatrinho, fantoches, mímica ou jogo de tabuleiro para imitar o que inevitavelmente teríamos cara a cara."

. Método que consolida o aprendizado:

"Eu nunca tinha tido a oportunidade de debater esses temas diretamente com pessoas que representam esses futuros pacientes, e isso faz toda a diferença. É um aprendizado mais sólido, consistente e proveitoso, se comparado a meras discussões sobre o tema, sem que haja a participação de pessoas representantes do grupo enfocado, no caso, os surdos." 


\section{Discussão}

A mudança de percepção da pessoa surda a partir da falta, ou do que se pensa faltar a ela, para alguém sendo visto como diferente dos outros apenas por não ouvir, de acordo com os resultados apresentados, parece resultar da presença de tutores surdos e do espaço aberto ao diálogo entre estes e os estudantes. Quando não é oferecida ao estudante de medicina a oportunidade de aprender a se comunicar com pessoas surdas, é como se essas pessoas não existissem. Mesmo quando encontram pacientes surdos em estágios e plantões, a tendência, de acordo com este conjunto de dados, parece ser a reprodução de equívocos já descritos, com todos os seus riscos, por falta de uma comunicação eficiente. Através dos relatos dos estudantes, ficou claro que se sentiram mais seguros e menos desconfortáveis com a possibilidade de atenderem pessoas surdas, a partir do que aprenderam em curtíssimo tempo. Os estudantes se mostraram receptivos à inclusão de temas ligados a pessoas com deficiência no currículo de graduação em medicina, sugerindo, inclusive, que essa inclusão não fosse pontual, com o que concordamos.

Interessante também foi terem percebido que as dificuldades de comunicação encontradas no atendimento da pessoa surda são semelhantes às que surgem na anamnese de pacientes estrangeiros que não conhecem a língua portuguesa. Durante a discussão em aula, uma das alunas citou o exemplo de uma mulher que procurou atendimento para seu filho na Unidade de Saúde. Recém-chegada de um país asiático, não sabia português, e a dificuldade de comunicação foi muito semelhante à encontrada com pessoas surdas, percebendo a aplicação das habilidades adquiridas, também, a outros grupos de pacientes.

A única crítica negativa presente nas avaliações dos estudantes foi quanto ao número de estudantes em sala durante a dinâmica. Por conta da disponibilidade dos profissionais do NOSS de participarem de apenas uma atividade por semestre, não foi possível dividir a turma, o que teria sido o ideal.

\section{Conclusão}

As escolas médicas não deveriam mais formar profissionais que desconhecessem as necessidades de saúde de pessoas com deficiência, as formas alternativas de comunicação com pessoas não verbais, e os riscos que essa lacuna pode gerar. Atividades simples como as aqui apresentadas, buscando não apenas sensibilizar os estudantes, mas muni-los com conhecimentos e habilidades de comunicação com a pessoa surda, podem contribuir para mudar o cenário atual. A participação de pessoas surdas foi altamente valorizada pelos estudantes. Esperamos que essa experiência possa inspirar a implantação, em outras escolas médicas, de iniciativas semelhantes.

A promoção e sustentação da inclusão de temas relativos às pessoas com deficiência, infelizmente, ainda dependem de iniciativas individuais, e não de uma política das escolas médicas que pudesse garantir sua continuidade. No segundo semestre de 2010, constatamos essa fragilidade, com a interrupção da oferta deste conteúdo por afastamento da coordenadora da disciplina Saúde e Sociedade III, que partiu para a busca por parcerias com professores de outras disciplinas do curso médico. 


\section{Colaboradores}

As autoras trabalharam juntas em todas as etapas de produção do manuscrito.

\section{Referências}

ALMEIDA, R.C.N. Sinalizando a sexualidade: uma proposta pedagógica de intervenção na comunidade surda. 2007. Dissertação (Mestrado) - Faculdade de Educação. Universidade Plínio Leite, Niterói. 2007.

BARDIN, L. Análise de conteúdo. Trad. Luís Antero Reto; Augusto Pinheiro. Lisboa: Edições 70, 1977.

BARNETT, S. Cross-cultural communication with patients who use American Sign Language. Fam. Med., v.34, n.5, p.376-82, 2002.

BAT-CHAVA, Y.; MARTIN, D.; KOSCIW, J.G. Barriers to HIV/Aids knowledge and prevention among deaf and hard of hearing people. AIDS Care, v.17, n.5, p.623-34, 2005.

BRASIL. Ministério da Educação. Resolução CNE/CES n 4, de 7 de novembro de 2001. Diário Oficial da União, Brasília, DF, 9 nov. de 2001. Seção 1, p. 38.

Ministério da Saúde. Secretaria de Atenção à Saúde. Departamento de Ações Programáticas Estratégicas. A pessoa com deficiência e o Sistema Único de Saúde. 2.ed. Brasília: MS, 2006.

Política Nacional de Saúde da Pessoa com Deficiência, instituída através da Portaria n 1.060, de 5 de junho de 2002. Diário Oficial da União Brasília, DF, 10 jun. 2002

BYRON, M. et al. What does 'disability' mean for medical students? An exploration of the words medical students associate with the term 'disability'. Med. Educ., v.39, n.2, p.176-83, 2005

BYRON, M.; DIEPPE, P. Educating health professionals about disability: 'attitudes, attitudes, attitudes'. J. R. Soc. Med., v.93, n.8, p.397-8, 2000.

CHAVEIRO, N. Encontro do paciente surdo que usa língua de sinais com os profissionais da saúde. 2007. Dissertação (Mestrado) - Programa de Pós-Graduação em Ciências da Saúde, Universidade Federal de Goiás, Goiânia. 2007.

CHELINI, P. et al. A contribuição do tema deficiência/pessoa com deficiência na formação médica: o olhar dos estudantes de medicina. In: CONGRESSO BRASILEIRO DE EDUCAÇÃO MÉDICA, 44., 2006, Gramado. Anais... Gramado, 2006. p.337.

CONILL, A. Living with disability: a proposal for medical education. J. Am. Med. Assoc., v.279, n.1, p.83, 1988.

COSTA, L.S.M. Deficiência e educação médica. In: CONGRESSO BRASILEIRO DE EDUCAÇÃO MÉDICA, 43., 2005, Natal. Anais... Natal, 2005. p.556.

COSTA, L.S.M.; BOTELHO, I.V.; SOUZA, L.S. Abordagem do tema deficiência na literatura médica. In: CONGRESSO BRASILEIRO DE EDUCAÇÃO MÉDICA, 46., 2008, Salvador. Anais... Salvador, 2008. p.246.

COSTA, L.S.M. et al. O atendimento em saúde através do olhar da pessoa surda: avaliação e propostas. Rev. Bras. Clín. Méd., v.7, n.3, p.166-70, 2009.

DUGGAN, A. et al. What can I learn from this interaction? A qualitative analysis of medical student self-reflection and learning in a standardized patient exercise about disability. J. Health Commun., v.14, n.8, p.797-811, 2009.

EDDEY, G.E.; ROBEY, K.L. Considering the culture of disability in cultural competence education. Acad. Med., v.80, n.7, p.706-12, 2005. 
FELIPE, T.A. A Língua Brasileira de Sinais - Libras. In: (Org.). Libras em contexto: curso básico - livro do estudante. 5.ed. Rio de Janeiro: Libras Editora Gráfica, 2005. p.21-6.

FERREIRA, C.L.; KOIFMAN, L.; COSTA, L.S.M. Relação médico-paciente e a perspectiva inclusiva da pessoa com deficiência In: CONGRESSO BRASILEIRO DE EDUCAÇÃO MÉDICA, 43., 2005, Natal. Anais... Natal, 2005. p.570.

FILGUEIRAS, S.L.; DESLANDES, S.F. Avaliação das ações de aconselhamento: análise de uma perspectiva de prevenção centrada na pessoa. Cad. Saude Publica, v.15, supl.2, p.121-31, 1999.

GOMES, R.A. Análise de dados em pesquisa qualitativa. In: MINAYO, M.C.S. (Org.). Pesquisa social: teoria, método e criatividade. 7.ed. Petrópolis: Vozes, 1997. p.67-80.

HENLEY, L.D. A home visit programme to teach medical students about children with special needs. Med. Educ., v.33, n.10, p.749-52, 1999.

IEZZONI, L.I. et al. Communicating about health care: observations from persons who are deaf or hard of hearing. Ann. Int. Med., v.140, n.5, p.356-62, 2004.

MARGELLOS-ANAST, H.; ESTARZIAU, M.; KAUFMAN, G. Cardiovascular disease knowledge among culturally deaf patients in Chicago. Prevent. Med., v.42, n.3, p.235-9, 2006.

MARTIN, H.L. et al. Cerebral palsy: what do medical students know and believe? J. Pediatr. Child Health, v.41, n.1-2, p.43-7, 2005.

MINIHAN, P.M. et al. Teaching about disability: Involving patients with disabilities as medical educators. Disab. Stud. Quart., v.24, n.4, 2004. Disponível em:

<http://www.dsq-sds.org/article/view/887/1062> . Acesso em: 18 ago. 2011.

MITCHELL, K.R. et al. An investigation of the attitudes of medical students to physically disabled people. Med. Educ., v.18, n.1, p.21-3, 1984.

PAULA, A.R. Comentário ao artigo 3 da Convenção sobre os Direitos das Pessoas com Deficiência: princípios gerais. In: RESENDE, A.P.C.; VITAL, F.M.P. (Orgs.). A Convenção sobre os Direitos das Pessoas com Deficiência comentada. Brasília: Secretaria Especial dos Direitos humanos. Coordenadoria Nacional para Integração da Pessoa Portadora de Deficiência, 2008. p.30-2.

ROSSI, O.S.; BATISTA, N.A. O ensino de comunicação na graduação em Medicina uma abordagem. Interface - Comunic., Saude, Educ., v.10, n.19, p.93-102, 2006.

SABHARWAL, S.; BROWNELL, A.M. Objective assessment and structured teaching of disability etiquette. Acad. Med., v.76, n.5, p.509, 2001.

SABHARWAL, S.; SEBASTIAN, J.L.; LANOUETTE, M. An educational intervention to teach medical students about examining disabled patients. J. Am. Med. Assoc., v.284, n.9, p.1080-1, 2000.

SASSAKI, R.K. Nada sobre nós sem nós: da integração à inclusão. Rev. Nac. Reabil., v.10, n.57, p.8-16, 2007.

SILVA, N.C.Z.; COSTA, L.S.M. Desenvolvendo habilidades de comunicação dos estudantes de medicina com pessoas surdas e com dificuldade de articulação da fala: relato de experiência. In: CONGRESSO BRASILEIRO DE EDUCAÇÃO MÉDICA, 46., 2008, Salvador. Anais... Salvador, 2008. p.64.

STEINBERG, A.G. et al. Deaf women: experiences and perceptions of health system access. J. Women Health, v.11, n.8, p.729-41, 2002.

SYMONS, A.B.; MCGUIGAN, D.; AKL, E.A. A curriculum to teach medical students to care for people with disabilities: development and initial implementation. BMC Med. Educ., v.9, n.78, 2009. Disponível em: <http://www.biomedcentral.com/1472-6920/ 9/78>. Acesso em: 13 jul. 2011. 
THISTLETHWAITE, J.E.; EWART, B.R. Valuing diversity: helping medical students explore their attitudes and beliefs. Med. Teach., v.25, n.3, p.277-81, 2003.

TRACY, J.; IACONO, T. People with developmental disabilities teaching medical students - does it make a difference? J. Intellect. Dev. Disabil., v.33, n.4, p.345-8, 2008.

UBIDO, J.; HUNTINGTON, J.; WARBURTON, D. Inequalities in access to healthcare faced by women who are deaf. Health Soc. Care Commun., v.10, n.4, p.247-53, 2002. doi: 10.1046/j.1365-2524.2002.00365.x

UNITED STATES OF AMERICA. Department of Justice. Communicating with people who are deaf or hard of hearing in hospital setting. Civil Rights Division. Americans with disability act business brief. Disability Rights Section. Washington: ADA, 2003. p.1-4. Disponível em: <http://www.ada.gov/hospcombrscr.pdf>. Acesso em: 13 out 2010.

VIEIRA, V.H.R. et al. Atenção integral à saúde da pessoa com deficiência: a teoria na prática. In: CONGRESSO BRASILEIRO DE EDUCAÇÃO MÉDICA, 46., 2008, Salvador. Anais... Salvador, 2008. p.65.

VLAK, T. et al. Teaching disability and rehabilitation medicine at the medical school in Split, Croatia. Croatian Med. J., v.45, n.1, p.99-102, 2004.

World Health Organization. World report on disability 2001. Disponível em: <http://www.who.int/disabilities/world_report/2011/en>. Acesso em: 10 jun. 2011.

ZAZOVE, P.; DOUKAS, D.J. The silent health care crisis: ethical reflections of health care for deaf and hard-of-hearing persons. Fam. Med., v.26, n.6, p.387-90, 1994.

Desconhecer as pessoas surdas, não possuir habilidades de comunicação e apresentar atitudes negativas em relação a elas pode trazer riscos para a saúde desses pacientes. Descreve-se a experiência de inclusão do tema surdez em disciplina dirigida a estudantes da Universidade Federal Fluminense. Durante duas aulas da disciplina Saúde e Sociedade III, de 2007 a 2010, 553 estudantes de medicina do segundo ano foram expostos a leituras, discussões, vídeo e vivências com participação de pessoas surdas. Foram solicitadas avaliações livres e anônimas. A análise das avaliações revelou temas contemplando a aquisição de conhecimentos, habilidades de comunicação e atitudes positivas em relação a pacientes surdos. $O$ contato direto com pessoas surdas mostrou-se fundamental para a promoção de atitudes positivas. É importante não apenas sensibilizar os estudantes de medicina, mas instrumentalizá-los para um atendimento que garanta a autonomia e independência de pacientes surdos.

Palavras-chave: Educação médica. Pessoas surdas. Habilidades de comunicação. 


\section{Developing medical students' attitudes, knowledge and skills in healthcare for deaf people}

Not knowing deaf people, lacking communication skills and presenting negative attitudes towards them may lead to risks to these patients' health. An inclusion experience on the topic of deafness in an academic discipline directed towards students at the Fluminense Federal University is described here. During two classes in the module Health and Society III, from 2007 to 2010, 553 second-year medical students were exposed to readings, discussions, video and experiences with participation by deaf people. Freeform anonymous evaluations were requested. Analysis on these evaluations revealed themes relating to acquisition of knowledge, communication skills and positive attitudes towards deaf patients. Direct contact with deaf people was shown to be essential for promoting positive attitudes. It is important not only to sensitize medical students, but to provide them with tools to ensure deaf patients' autonomy and independence.

Keywords: Medical education. Deaf. Communication skills.

Desarrollo de actitudes, conocimientos y habilidades de los estudiantes de medicina en la atención en salud de las personas sordas

Desconocer las personas sordas, dejar de tener habilidades de comunicación y presentar actitudes negativas con relación a ellas puede generar riesgos para su salud. Es descrita la experiencia de inclusión del tema sordera en materia ofrecida para estudiantes de la Universidad Federal Fluminense, Brasil. Durante dos clases de Salud y Sociedad III, entre 2007 y 2010, 553 estudiantes de medicina del segundo año fueran expuestos a lecturas, discusiones, vídeo y experiencias con participación de personas sordas. Los alumnos evaluaran libremente y de forma anónima las actividades vividas. El análisis de las evaluaciones demostró que hubo adquisición de conocimiento, habilidades de comunicación y actitudes positivas con relación a pacientes sordos. El contacto directo con personas sordas se presentó fundamental para la promoción de actitudes positivas. Además de sensibilizar a los estudiantes, es necesario instrumentalizarlos para la atención en salud que garantice la autonomía e independencia de pacientes sordos.

Palabras clave: Educación médica. Personas surdas. Habilidades de comunicación. 


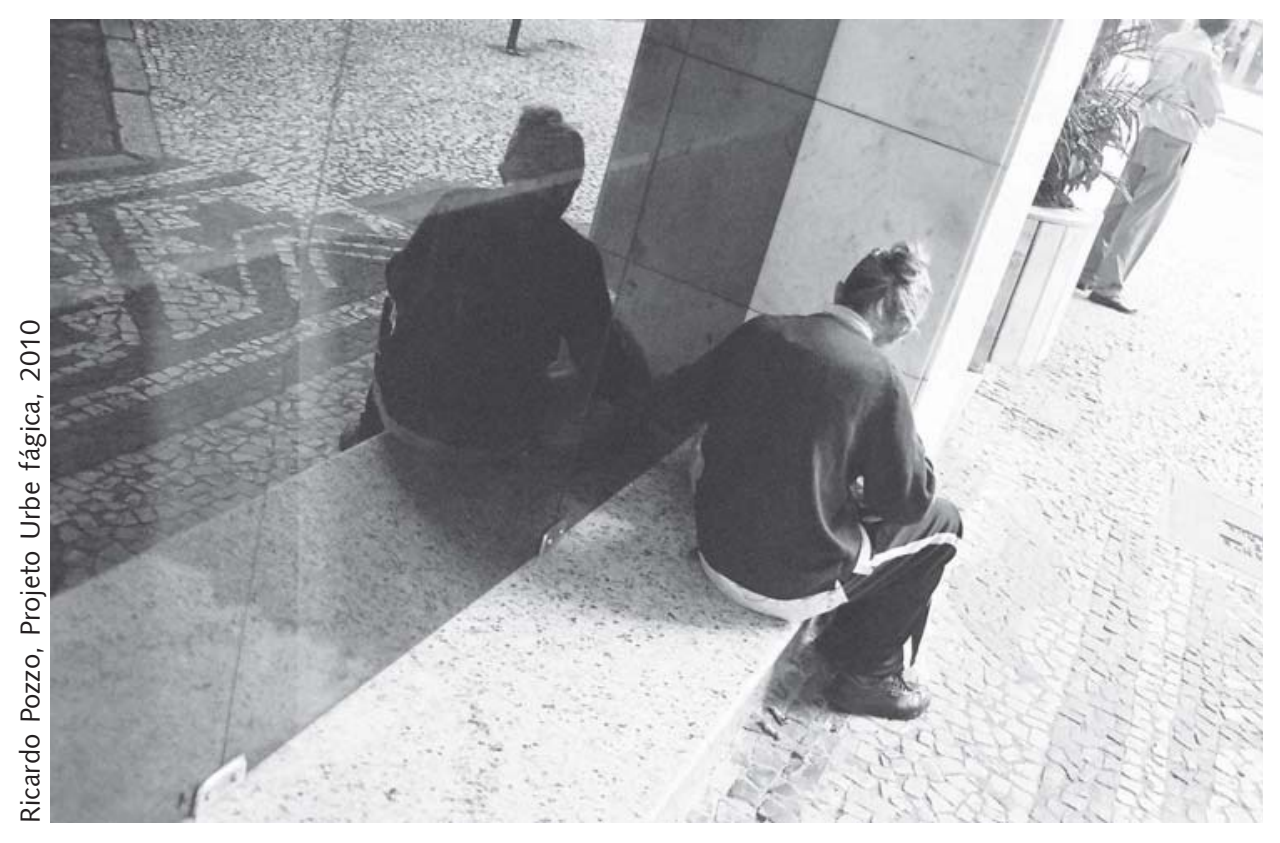

